\title{
BMJ Open Glycaemic control efficacy of oral antidiabetic drugs in treating type 2 diabetes: a protocol for network meta-analysis
}

\author{
Yongliang Jia, ${ }^{1}$ Yaoguang Lao, ${ }^{1}$ Siu-wai Leung ${ }^{1,2}$
}

To cite: Jia Y, Lao Y, Leung S-w. Glycaemic control efficacy of oral antidiabetic drugs in treating type 2 diabetes: a protocol for network meta-analysis. BMJ Open 2015;5:e006139. doi:10.1136/bmjopen-2014006139

- Prepublication history and additional material is available. To view please visit the journal (http://dx.doi.org/ 10.1136/bmjopen-2014006139).

YJ and YL are co-first authors.

Received 17 July 2014 Revised 16 October 2014 Accepted 6 November 2014

CrossMark

\footnotetext{
${ }^{1}$ State Key Laboratory of Quality Research in Chinese Medicine, Institute of Chinese Medical Sciences, University of Macau, Macao, China ${ }^{2}$ School of Informatics, University of Edinburgh, Edinburgh, UK
}

Correspondence to Dr Siu-wai Leung; siu@inf.ed.ac.uk

\begin{abstract}
Introduction: Past studies of network meta-analysis focused on evaluating drug combinations in treating type 2 diabetes but not on evaluating antidiabetic drugs in monotherapy. Clinical guidelines (eg, NICE (National Institute of Health and Care Excellence) clinical guidelines 66 and 87 ) were based only on the findings of individual clinical trials and pairwise meta-analysis in evaluating monotherapy. This study aims to fill this gap of research by conducting a Bayesian network metaanalysis to compare major antidiabetic drugs, including metformin, glimepiride, glyburide, glipizide, repaglinide, nateglinide, sitagliptin, vildagliptin, saxagliptin and SGLT-2 (sodium-glucose transporter-2) inhibitors.
\end{abstract}

Methods and analyses: Randomised controlled trials (RCTs) on the drug therapy of type 2 diabetes with outcome measures including glycosylated haemoglobin or fasting blood glucose will be included. The quality of included RTCs will be evaluated according to the Cochrane Collaboration's risk of bias tool. Traditional pairwise meta-analysis and Bayesian network metaanalysis will be conducted to compare the efficacies of antidiabetic drugs. Sensitivity analysis on the sample size of RCTs, meta-regression analysis on the follow-up periods, dosages and baselines of outcome measure, contradiction analysis between pairwise and network meta-analyses, and publication bias analysis, will be performed.

Ethics and dissemination: Ethical approval is not required because this study includes no confidential personal data and interventions on the patients.

Pairwise and network meta-analyses are based on the published RCT reports of eligible drugs in treating type 2 diabetes. The results of this study will be disseminated by a peer-reviewed publication.

Protocol registration number: PROSPERO CRD42014010567.

\section{INTRODUCTION}

Glycaemic control would prevent microvascular and macrovascular complications of type 2 diabetes. ${ }^{12}$ Several categories of oral antidiabetic drugs including biguanides, thiazolidinediones, sulfonylureas, meglitinides, DPP-4 (dipeptidyl peptidase-4) inhibitors and
Strengths and limitations of this study

- Network meta-analysis together with sensitivity analysis, contradiction analysis and publication bias analysis will evaluate the efficacies of multiple antidiabetic drugs.

- This study will provide evidence for clinical decision-makers to formulate better treatment of type 2 diabetes.

- This study is inherently retrospective and based on the published randomised controlled trails only.

$\alpha$-glucosidase inhibitors are available for monotherapy of type 2 diabetes. Efficacies of these drugs should be monitored for postmarketing evaluation and for updating of clinical guidelines. However, the latest National Institute for Health and Care Excellence (NICE) guidelines ${ }^{3}{ }^{4}$ for treating type 2 diabetes only included those randomised control trials (RCTs) and their meta-analyses published before 2010 .

Even if the clinical guidelines were up to date, there are still gaps to be filled among the current pieces of evidence for the glycaemic control efficacy of oral antidiabetic drugs. First, the current evidence for oral antidiabetic drug efficacies was only limited to a number of head-to-head RCTs and meta-analyses, including the most comprehensive study by the Agency for Healthcare Research and Quality, ${ }^{5}$ and does not cover all possible comparisons among individual drugs. In this situation, network meta-analysis (NMA) that can integrate the evidence from direct and indirect comparisons ${ }^{6}$ would be applicable. Second, efficacy ranking of the oral antidiabetic drugs was still unknown. The drug recommendation by clinical guidelines was not based on comprehensive and systematic studies for comparing multiple drugs. This gap also suggests an imminent need for NAM that can rank all evaluated interventions. $^{7}$ 
While NAM was used in comparing the efficacies of oral antidiabetic drugs, the available network meta-analyses ${ }^{8-10}$ evaluated only treatments combined with metformin. The monotherapy efficacies of individual drugs have not been studied by NAM.

This study conducted a Bayesian NAM ${ }^{5} 11$ to compare the glycaemic control efficacy of popular oral antidiabetic drugs, including metformin, glimepiride, glyburide, glipizide, repaglinide, nateglinide, sitagliptin, vildagliptin, saxagliptin and SGLT-2 (sodium-glucose transporter-2) inhibitors.

\section{OBJECTIVE}

The objective of this study is to compare efficacies of popular antidiabetic drugs by Bayesian NAM on RCTs.

\section{METHODS AND ANALYSIS \\ Design}

Systematic review and Bayesian NAM.

\section{Information sources}

Clinical trial reports will be searched from PubMed and Cochrane Library.

\section{Search strategies}

Drug names, synonyms of type 2 diabetes (eg, type 2 diabetes, type II diabetes and non-insulin-dependent diabetes) and "random*" will be used as keywords to search titles or abstracts for eligible RCTs from major databases including PubMed, Cochrane Library, ScienceDirect and EMBASE, as well as Food and Drug Administration medical reviews and clinicaltrials.gov website. The search is scheduled between August and October in 2014. For example, the following search strategy will be used in searching PubMed:

1. metformin

2. type 2 diabetes

3. random*

4. 1 in title or abstract

5. 2 in title or abstract

6. 3 in title or abstract

7. 4 and 5 and 6

\section{Eligibility criteria}

The retrieved reports will be screened according to the checklist of eligibility (see online supplementary appendix 1) and the eligibility criteria shown below including participants, interventions, controls, types of study and other criteria.

\section{Participants}

Inclusion: The participants must be adults, aged at least 18 years, suffering from and requiring treatment for type 2 diabetes. Exclusion: The participants suffering from other diabetic disease conditions or aged under 18 years.

\section{Interventions}

Inclusion: Any RCT that evaluates the efficacy of these drugs. Exclusion: Any RCT that evaluates other drugs or combined treatments of multiple drugs or placebo.

\section{Controls}

Inclusion: Any RCT that evaluates the efficacy of these drugs other than the drug of intervention or placebo. Exclusion: Any RCT that evaluates other drugs or combined treatments of multiple drugs.

Types of study

Inclusion: Only RCTs will be included. Exclusion: Observational cohort and case-control studies, case reports, experimental studies and reviews will be excluded.

\section{Other criteria}

Other inclusion criteria: The RCTs must report complete efficacy data of glycosylated haemoglobin (HbAlc) or fasting blood glucose (FPG) of each treatment. Follow-up periods or durations in RCTs are at least 4 weeks. Other exclusion criteria are (1) duplicated or redundant studies and (2) combined treatments with multiple drugs.

\section{Study selection}

Reviewers will screen all titles or abstracts or full texts for database records independently according to the eligibility criteria. Disagreements between reviewers will be resolved by consensus. Selection process of relevant studies retrieved from databases will be shown in a PRISMA-compliant ${ }^{12}$ flow chart (figure 1).

\section{Data extraction and quality assessment}

Data of the study characteristics and the clinical outcome measures will be extracted. The data extracted from the RCTs are: (1) authors; (2) publication year; (3) baseline of outcome measures; (4) sample sizes; (5) interventions of both arms; (6) dosages of both arms and (7) treatment outcome measures including HbAlc and FPG. The data will be standardised (table 1). The quality of eligible studies will be evaluated according to the Cochrane Collaboration's risk of bias tool for assessing risk of bias (table 2) ${ }^{13}$ Radar chart (or star chart) ${ }^{14}$ will be used to summarise the results.

\section{Outcome measures}

Outcome measures of antidiabetic efficacy include mean changes of HbAlc (primary outcome) and FPG (secondary outcome) from baseline and their corresponding variation.

\section{Statistical analysis}

Pairwise meta-analysis of the included RCTs with random effect model ${ }^{15}{ }^{16}$ due to the expected heterogeneity will be conducted. Mean difference (MD) will be used to synthesis the continuous outcome data: mean changes from baseline of the HbAlc (\%) and FPG $(\mathrm{mol} / \mathrm{L})$ in both arms. $\mathrm{I}^{2}$ was used to estimate the 
Figure 1 Flow chart of the study selection (RCT, randomised controlled trial).

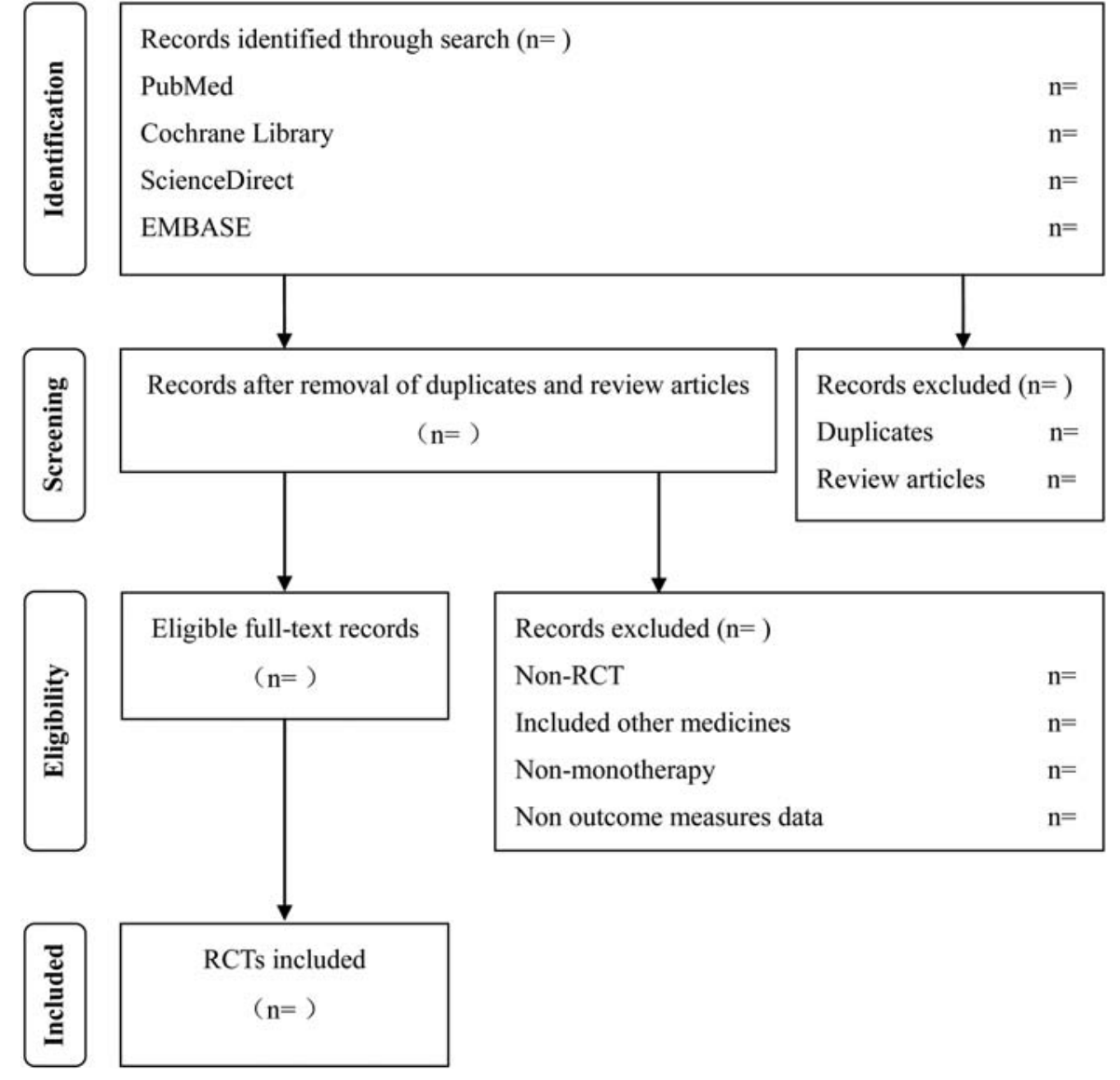

heterogeneity. ${ }^{17}$ Networks will be generated to visualise the results of pairwise meta-analysis and the current evidence from the included RCTs.

NAM based on the Bayesian hierarchical model ${ }^{8}$ will be performed to compare the efficacy of selected drugs. Placebo will be used as common comparison ${ }^{18}$ in NMA. Relative MD to the placebo will be output to assess the efficacy. The probability of each drug being ranked in each position based on HbAlc will be computed. ${ }^{19}$ Kendall's test will be used to test the correlation between the relative MD and the ranking position.

Sensitivity analysis based on the sample size of the RCTs will be conducted when RCTs with sample size less than 50 are excluded. Sensitivity analysis will also be conducted on different baselines. Meta-regression analyses will be conducted on the different follow-up periods and dosages for drugs of the included RCTs. Begg's ${ }^{20}$ and Egger's tests ${ }^{21}$ will be used to evaluate the publication bias. Agreement will be computed to assess the consistency between pairwise and network meta-analyses.

R software ${ }^{22}$ will be used to implement the analysis workflow. Package "metafor" 23 will be used to conduct pairwise meta-analysis. Package "igraph" ${ }^{24}$ will be used to visualise the networks. Package " $f m s b$ " 25 will be used to visualise the results of risk of bias assessment. Package 'GeMTC', ${ }^{26}$ 'R2WinBUGS' ${ }^{27}$ in $\mathrm{R}$ and WinBUGS' ${ }^{28}$ will be used to conduct NAM. Package 'ggplot2'29 will be used to visualise the distribution of ranking probability distribution. $p$ Values lower than 0.05 will be considered statistically significant.

\section{ETHICS AND DISSEMINATION \\ Ethical issues}

No ethical approval is required because this study includes no confidential personal data or interventions with the patients.

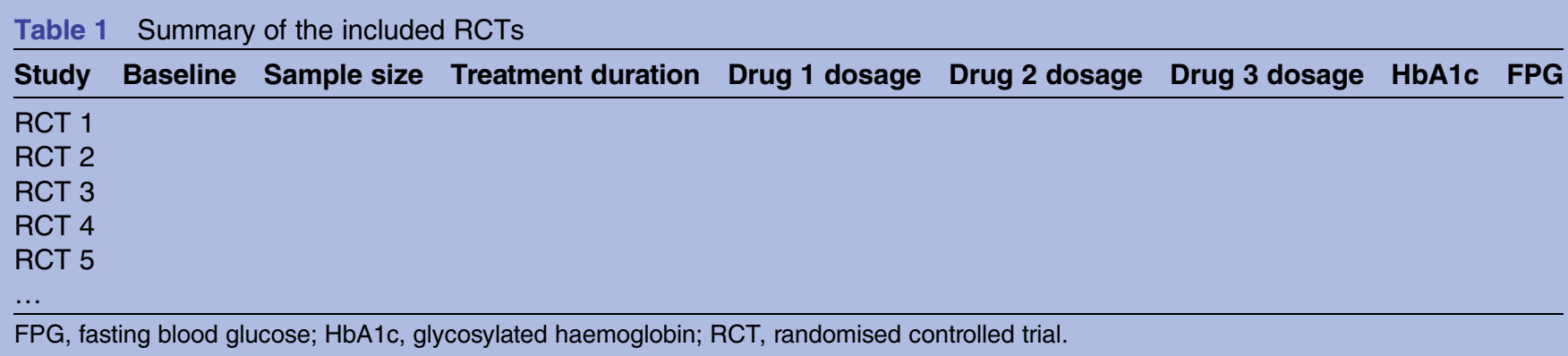


Table 2 Randomised controlled trial (RCT) quality assessment according to the Cochrane Collaboration's risk of bias tool

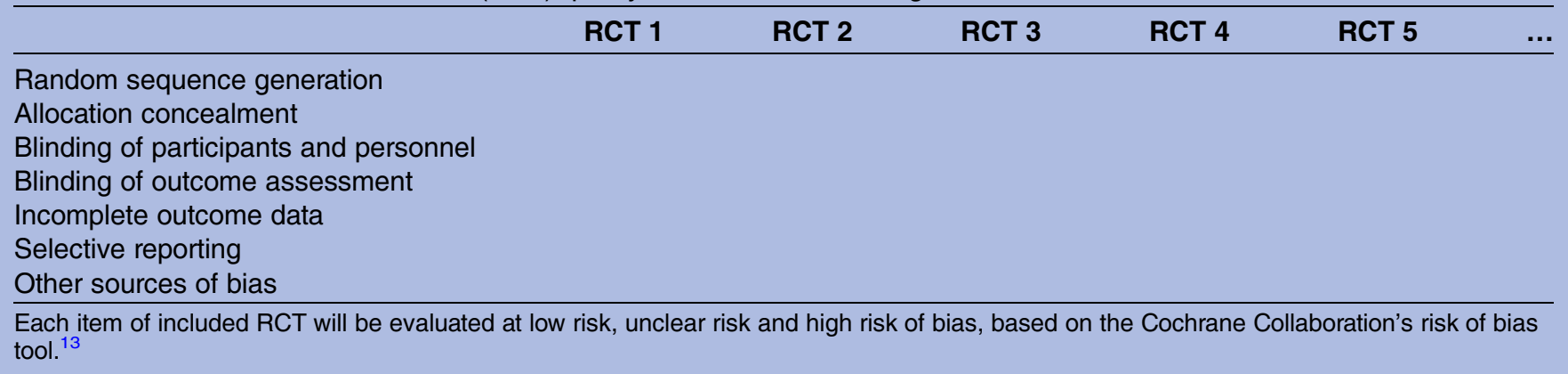

\section{Publication plan}

This protocol has been registered (Registration number: CRD42014010567) with the PROSPERO (International Prospective Register of Systematic Reviews). ${ }^{30}$ The procedures of this systematic review and NAM will be conducted in accordance with the PRISMA-compliant guideline. The results of this systematic review and NAM will be submitted to a peer-reviewed journal for publication.

Contributors S-wL conceived the study. S-wL, YL and YJ designed the protocol. YL and YJ tested the feasibility of the study. YJ, YL and S-wL wrote the protocol and approved the final manuscript.

Funding The study is part of the 'Open systematic reviewing of clinical trials' project supported by a research grant (MYRG190-Y3-L3-ICMS11-LSW) received from the University of Macau.

Competing interests None.

Provenance and peer review Not commissioned; externally peer reviewed.

Open Access This is an Open Access article distributed in accordance with the Creative Commons Attribution Non Commercial (CC BY-NC 4.0) license, which permits others to distribute, remix, adapt, build upon this work noncommercially, and license their derivative works on different terms, provided the original work is properly cited and the use is non-commercial. See: http:// creativecommons.org/licenses/by-nc/4.0/

\section{REFERENCES}

1. Holman RR, Paul SK, Bethel MA, et al. 10-Year follow-up of intensive glucose control in type 2 diabetes. $N$ Engl $J$ Med 2008;359:1577-89.

2. Turner RC, Cull CA, Frighi V, et al. Glycemic control with diet, sulfonylurea, metformin, or insulin in patients with type 2 diabetes mellitus: progressive requirement for multiple therapies (UKPDS 49). UK Prospective Diabetes Study (UKPDS) Group. JAMA 1999;281:2005-12.

3. National Institute for Health and Clinical Excellence. Type 2 diabetes: national clinical guideline for management in primary and secondary care (update) (partially updated by CG87). NICE clinical guideline 66.

4. National Institute for Health and Clinical Excellence. Type 2 diabetes: the management of type 2 diabetes. NICE clinical guideline 87.

5. Bennett W, Wilson L, Bolen S. Oral diabetes medications for adults with type 2 diabetes: an update. Agency for Healthcare Research and Quality $2011 ; 1-320$.

6. Lu G, Ades AE. Combination of direct and indirect evidence in mixed treatment comparisons. Stat Med 2004;23:3105-24.

7. Salanti G, Ades AE, loannidis JP. Graphical methods and numerical summaries for presenting results from multiple-treatment metaanalysis: an overview and tutorial. J Clin Epidemiol 2011;64:163-71.

8. Gross JL, Kramer CK, Leitão CB, et al. Effect of antihyperglycemic agents added to metformin and a sulfonylurea on glycemic control and weight gain in type 2 diabetes: a network meta-analysis. Ann Intern Med 2011;154:672-9.
9. Liu S, Tu Y, Chien M, et al. Effect of antidiabetic agents added to metformin on glycaemic control, hypoglycaemia and weight change in patients with type 2 diabetes: a network meta-analysis. Diabetes Obes Metab 2012;14:810-20.

10. Goring S, Hawkins N, Wygant G, et al. Dapagliflozin compared with other oral anti-diabetes treatments when added to metformin monotherapy: a systematic review and network meta-analysis. Diabetes Obes Metab 2014;16:433-42.

11. Jansen JP, Crawford B, Bergman G, et al. Bayesian meta-analysis of multiple treatment comparisons: an introduction to mixed treatment comparisons. Value Health 2008;11:956-64.

12. Moher D, Liberati A, Tetzlaff J, et al. Preferred reporting items for systematic reviews and meta-analyses: the PRISMA statement PLoS Med 2009;6:1-6.

13. Higgins JPT, Altman DG, Gøtzsche PC, et al. The Cochrane Collaboration's tool for assessing risk of bias in randomised trials. BMJ 2011;343:d5928.

14. Chambers JM, Cleveland WS, Tukey PA, et al. Graphical methods for data analysis. Wadsworth International Group, Duxbury Press, 1983.

15. Viechtbauer W. Bias and efficiency of meta-analytic variance estimators in the random-effects model. J Educ Behav Stat 2005;30:261-93.

16. Hedges LV, Vevea, JL. Fixed-and random-effects models in meta-analysis. Psychol Methods 1998;3:486-504.

17. Higgins JPT, Thompson SG, Deeks JJ, et al. Measuring inconsistency in meta-analyses. BMJ 2003;327:557-60.

18. Jansen JP, Fleurence R, Devine $B$, et al. Interpreting indirect treatment comparisons and network meta-analysis for health-care decision making: report of the ISPOR Task Force on Indirect Treatment Comparisons Good Research Practices: part 1. Value Health 2011;14:417-28.

19. Cipriani A, Furukawa TA, Salanti G, et al. Comparative efficacy and acceptability of 12 new-generation antidepressants: a multiple-treatments meta-analysis. Lancet 2009;373:746-58.

20. Begg CB, Mazumdar M. Operating characteristics of a rank correlation test for publication bias. Biometrics 1994;50: 1088-101.

21. Egger M, Davey Smith G, Schneider M, et al. Bias in meta-analysis detected by a simple, graphical test. BMJ 1997;315:629-34.

22. R Development Core Team. $\mathrm{R}$ : a language and environment for statistical computing. Reference index version 2.12.0. R Foundation for Statistical Computing, Vienna, Austria. 2008.

23. Viechtbauer $\mathrm{W}$. Conducting meta-analyses in $\mathrm{R}$ with the metafor package. J Stat Softw 2010;36:1-48.

24. Csardi G, Nepusz T. The igraph software package for complex network research. Inter J Complex Syst 2006;1695.

25. Nakazawa M. Functions for medical statistics book with some demographic data. R package Version 0.4.5, 2014.

26. Van Valkenhoef G, Lu G, de Brock B, et al. Automating network meta-analysis. Res Synth Methods 2012;3:285-99.

27. Sturtz S, Ligges $U$, Gelman A. A package for running WinBUGS from R. J Stat Softw 2005;12:1-16.

28. Lunn DJ, Thomas A, Best N, et al. WinBUGS-a Bayesian modelling framework: concepts, structure, and extensibility. Stat Comput 2000;10:325-37.

29. Wickham H. ggplot2: Elegant Graphics for Data Analysis. Springer, 2009.

30. Jia Y, Lao Y, Leung S. Glycemic control efficacy of oral anti-diabetic drugs in treating type 2 diabetes: a protocol for network meta-analysis. PROSPERO 2014; CRD42014010567. 